\title{
MAPPING SPEM PROCESS SPECIFICATIONS TO ACTIVITY CYCLE DIAGRAMS
}

\author{
Pagliares, R. M. ${ }^{*, * *} \&$ Hirata, C. M." \\ *Computer Science Department - IEC - Instituto Tecnológico de Aeronáutica, ITA, \\ Pca. Mal. do Ar Eduardo Gomes, 50, 12228-900, São José dos Campos, SP, Brazil \\ ** Universidade Federal de Alfenas, UNIFAL-MG, Alfenas, MG, Brazil \\ E-Mail: pagliares@bcc.unifal-mg.edu.br,hirata@ita.br
}

\begin{abstract}
The development of discrete event simulation models to simulate engineering processes is a complex endeavour because it requires a great deal of effort, time and expertise in different areas, in addition to knowledge of techniques of modelling and implementation. We aim to provide a model-driven approach to automate the generation of simulation models from process models. The approach consists of algorithms that map elements of SPEM (Software \& Systems Process Engineering Metamodel) to XACDML (eXtensible Activity Cycle Diagrams Markup Language). SPEM is a notation used to model engineering processes. XACDML is a textual specification of ACD. ACD is graphical notation to represent discrete event simulation models. We extended XACDML to cope with SPEM hierarchical elements enabling the mappings between the models. We developed a tool and use it in an example in order to demonstrate the feasibility of our approach. The results indicate that the approach is feasible and reduces the burden associated with building simulation models of engineering processes.

(Received in June 2017, accepted in November 2017. This paper was with the authors 1 week for 2 revisions.)
\end{abstract}

Key Words: Software \& Systems Process Engineering Metamodel (SPEM), Activity Cycle Diagrams (ACD), Automatic Model Generation, Discrete Event Simulation

\section{INTRODUCTION}

Process engineers and project managers are constantly monitoring and evaluating process performance in development projects in order to know its status and if the project goals will be achievable. The evaluation of process can give insights to potential process changes too. Proposing and evaluating process changes are generally complex tasks since they require knowledge of the process, inputs, available resources, used resources, and external factors that affect the project. Moreover, the task of evaluating process changes requires systematic approaches and techniques to support it. Discrete Event Simulation (DES) is one of the techniques. However, the development of DES models of engineering processes is a complex endeavour $[1,2]$. It requires a great deal of effort, time and expertise in different areas, in addition to knowledge of techniques to model and implement simulators.

The goal is to provide a model-driven approach to automate the generation of DES models of SPEM process models. The approach uses SPEM [3] as notation for defining engineering processes and a textual representation of Activity Cycle Diagrams (ACD) [4] named XACDML [5] to represent DES models. An engineering process (process for short) modelled with SPEM is translated into an extended XACDML model containing the structure, behaviour and quantitative parameters of the simulation. The approach supports the translation by applying mapping algorithms. We extended XACDML to deal with modelling elements found in processes but not defined in XACDML models, such as phases and milestones.

Our approach is materialized in a tool that generates simulation models allowing tailoring the generation process to fit analysts' interests. The tool enables experimentation of the generated XACDML simulation model by transforming it, on-the-fly, into reusable Java 
code. We employed the tool in a real project available in literature [6] as application of the approach.

The structure of this paper is as follows. In Section 2 we provide background on the notations used within our approach. Section 3 presents the related work. The approach uses mapping algorithms described in Section 4. In Section 5, we outline the tool support for the approach. Section 6 discusses the use of the tool with an example to demonstrate its applicability. Section 7 is devoted to discussions. We present concluding remarks in Section 8.

\section{BACKGROUND}

SPEM is a notation for modelling development processes. It is defined as a meta-model and as a UML profile [3] and has been used for authoring several processes used in engineering $[3,7]$. SPEM provides building blocks representing methods, life cycles, roles, activities, tasks, and work products used in systems engineering processes. Processes modelled with SPEM are hierarchical and represented by a Work Breakdown Structure (WBS). The process WBS is constituted of distinct Work Breakdown Elements (WBEs) such as phases, iterations, activities, milestones, and tasks. SPEM is the most used language for modelling processes, despite of existence of other languages [7].

ACD is a graphical notation to model DES systems. Its power relies on its simplicity, what makes it a useful tool to enable stakeholders' communication. ACD describes the behaviour of classes of entities as a cycle, which spans from their creation to their death. Every cycle is composed of the alternation of two kinds of states (active and dead) that are connected by directed arcs that indicate the path the entity should follow. The entity leaves the system upon completion of the cycle. Active states perform the business logic in the model and act upon entities, creating, moving, and changing their attributes. Their duration can be determined in advance by the use of appropriate probability distributions. Dead states are passive repositories of temporary entities and permanent entities (resources). They can be thought as states in which entities wait for something to occur.

XACDML [5] is an ACD compliant XML specification for DES, designed to allow exchange of models among tools. XACDML is a well-formed and valid XML language. Simulation analysts can tailor a model represented with XACDML for different purposes by specifying parameters for the simulation model such as probability distributions for active states, and capacity and initialization of dead states. XACDML may also be used as an intermediary language, being a starting point for other DES modelling formalisms.

\section{RELATED WORK}

We found several works on the automatic generation of simulation models in the literature. Most of them are targeted to specific domains, although there are some domain agnostic approaches. There are also a growing number of works that frame the problem of generating simulation models in the context of Model Driven Development (MDD).

Çetinkaya et al. [8] present a MDD framework that addresses model continuity at the different stages of Modelling and Simulation (M\&S) mirroring different models within a development process through transformation rules. The main goal of their work is to speed up the process of developing simulation models. The framework makes use of intermediate metamodels in addition to an executable simulation model. The authors suggest model transformations based on metamodels and the applicability of their framework is illustrated with a tool prototype.

Hollmann et al. [9] present CML-DEVS, a language that allows the representation of Discrete Event System Specification (DEVS) models in their abstract form in terms of 
mathematical and logical expressions. CML-DEVS allows a simulation analyst to define a simulation model even if he/she does not have programming skills or knowledge of a particular modelling language of a specific M\&S tool. CML-DEVS models are automatically transformed into the input models of different $M \& S$ tools through the use of transformation rules. The rules are implemented within a compiler in order to automate the transformations. The authors provide the transformation rules from DEVS to input models of DEVS-Suite [10] and PowerDEVS [11] tools.

Arias and Hirata [12] propose mappings of software models, annotated with performance information to a simulation model. Software models are based on UML deployment and state diagrams whereas the simulation model is represented with XACDML. The simulation model is translated to a simulation program for verification of performance requirements. The authors present an example in order to show the feasibility of the mappings.

To the best of our knowledge, the closest work to our current research is the work of Park et al. [2]. The authors propose an approach to derive a hybrid simulation model from a process model created with SPEM. The approach has steps that must be followed in order to derive a simulation model: mapping, modelling, and transforming. In the mapping step, the mapping between the elements of SPEM and DEVS-Hybrid is conducted. DEVS-Hybrid is an extension of DEVS formalism to the domain of hybrid software process simulation modelling. System Dynamics (SD) represents details concerning activity behaviours, and DES controls activity start/completion. In the modelling step, a process model is developed using a UML profile. The process model is transformed into a DEVS-Hybrid model by applying transformation rules in the transformation step. The authors also provide a tool to support their approach. In their approach, a simulation analyst must elaborate UML use cases to represent the WBS of a process and UML activity diagrams to represent the sequence of activities with input and output work products. Modelling these diagrams demands significant time from simulation analysts in order to transform the models, particularly in the absence of guidelines.

\section{MAPPING SPEM MODELS TO EXTENDED XACDML}

In this section, we describe how to map the elements of SPEM to elements of XACDML. SPEM provides some model elements that incorporate others (e.g. phase, iteration, and activity), allowing nesting of WBEs in a hierarchical fashion. Hierarchy cannot be modelled in XACDML, therefore, we extend it to artificially represent nested elements. Algorithms parameterized with quantitative data automate the mappings. Table I shows the algorithms' descriptions.

We apply the mapping algorithms in an example of a SPEM software process model that is translated into an extended XACDML simulation model to ease the understanding of the algorithms and XACDML extensions. Fig. 1 shows the process we use as example. It is a typical agile method for software development. The process has one phase named "software production" composed of several releases. Each release has the same structure. It includes a planning and development activity that starts with a release planning meeting where user stories are prepared to be implemented (prioritized, estimated, and split) and ends with several iterations of development in which developers perform the "pair programming with test driven development" task, before the next "planning and development" activity. For instance, the "pair programming with test driven development" task is performed by the developer role and has as input a user story that has been prepared during the "release planning meeting". The task produces an implemented user story as output. A milestone named "working software" is reached at the end of the software production phase. 
Table I: Mapping algorithms of SPEM elements to extended XACDML elements.

\begin{tabular}{|c|l|}
\hline Algorithm & \multicolumn{1}{c|}{ Description } \\
\hline$\# 1$ & Maps SPEM roles to XACDML permanent entities and resource dead states. \\
\hline$\# 2$ & $\begin{array}{l}\text { Maps SPEM work products to XACDML temporary entities, dead states, generate } \\
\text { active states, and destroy active states. }\end{array}$ \\
\hline$\# 3$ & $\begin{array}{l}\text { Maps SPEM tasks to XACDML activities. The mapping includes the new XML } \\
\text { element named <spem> and the new attributes named, spem_type, parent, } \\
\text { dependency_type, condition_to_process, processing_quantity, and } \\
\text { quantity_of_resources_needed. }\end{array}$ \\
\hline$\# 4$ & $\begin{array}{l}\text { Maps SPEM iterations, activities, phases, and milestones to dummy active states (i.e. } \\
\text { active states that when performed do not advance the simulation clock). The mapping } \\
\text { includes new attributes, such as timebox and iteration_behaviour. }\end{array}$ \\
\hline
\end{tabular}

We assume that processes may be quantifiable and data is available for adjusting the parameters of the XACDML model. XACDML models assume the specifications of the destinations of entities are made in an unequivocal manner. The SPEM process model of Fig. 1, after translation to XACDML, satisfies this assumption. We will refer back to this software process model in Section 6, where we experiment it with data taken from the literature.

\begin{tabular}{|c|c|c|c|c|c|}
\hline Presentation Name & Index & Predecessors & Model Info & Type & Optiona \\
\hline V Agile_process & 0 & & & DeliveryProcess & $\square$ \\
\hline$\nabla$ Software production & 1 & & & Phase & $\square$ \\
\hline $\mathbf{v}$ Release & 2 & & & Iteration & $\square$ \\
\hline $\mathbf{\nabla} 8$ Planning \& development & 3 & & & Activity & $\square$ \\
\hline v Release planning & 4 & & & TaskDescriptor & $\square$ \\
\hline User story & & & Mandatory Input & & $\square$ \\
\hline User story & & & Output & & $\square$ \\
\hline Customer & & & Primary Performer & & $\square$ \\
\hline Developer & & & Primary Performer & & $\square$ \\
\hline$\nabla$ Iteration & 5 & 4 & & Iteration & $\square$ \\
\hline v Pair programming with test driven development & 6 & & & TaskDescriptor & $\square$ \\
\hline User story & & & Mandatory Input & & $\square$ \\
\hline User story & & & Output & & $\square$ \\
\hline Developer & & & Primary Performer & & $\square$ \\
\hline 시 Working software & 7 & 1 & & Milestone & $\square$ \\
\hline
\end{tabular}

Figure 1: SPEM process for an agile method for software development.

After applying the mapping algorithms described in Table I, an XACDML model is generated (Fig. 2). Notice that its contents are an excerpt of the final XACDML. Line 01 identifies the model. The value of the $i d$ attribute is derived of the name of root node in Fig. 1. The acd element is "closed" in line 44. The execution of the algorithms, discussed in what follows, generates the remaining contents in Fig. 2.

Fig. 3 shows the first mapping algorithm in pseudo code. It works by parsing the SPEM process model of Fig. 1. The results of applying this algorithm in the example can be seen in lines 2 and 4 in Fig. 2. Algorithm \#1 receives as parameter an instance of DeliveryProcess (line 1). In SPEM, a DeliveryProcess represents the root node of the WBS representing the process. A SPEM role defines a set of related skills, competencies, and responsibilities of a set of individuals. For each SPEM role within the DeliveryProcess, an XACDML permanent entity is created and added to the output XACDML (lines 2-4). The statement at line 5 translates each SPEM role into its own resource dead state. Lines 6 and 7 of Fig. 3 allow simulation analysts to define the parameters for the type and capacity of the dead state to be generated and to include the dead state in the output XACDML. 


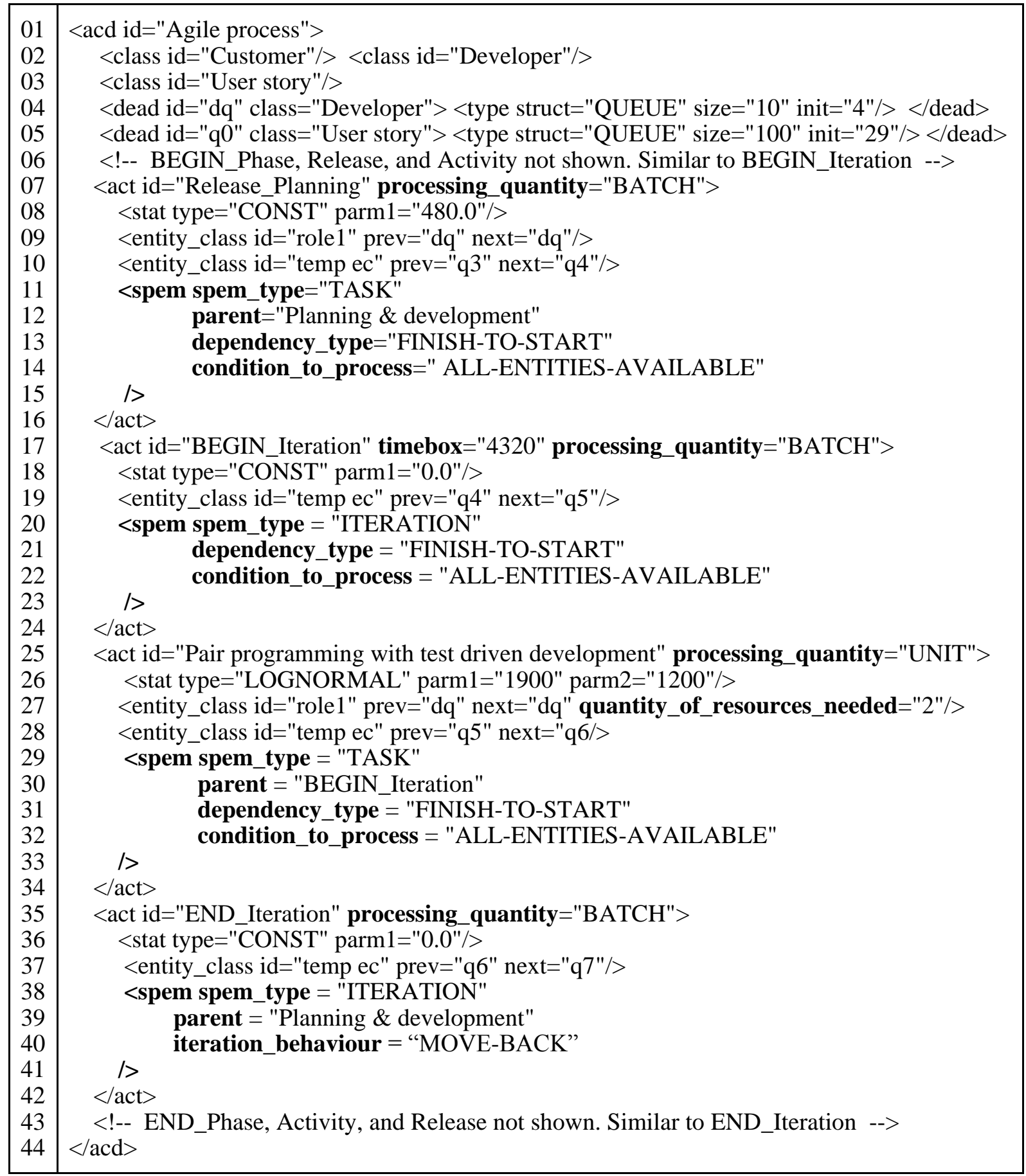

Figure 2: Excerpt of the XACDML simulation model generated after applying the algorithms discussed in this section. Extensions made to XACDML are in boldface.

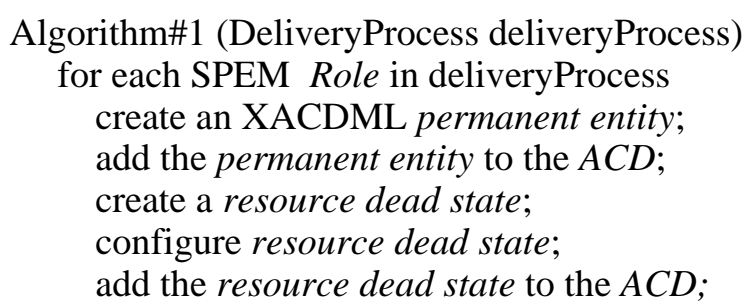

Figure 3: Mapping algorithm \#1 - "Maps SPEM roles to XACDML permanent entities and resource dead states". 
Fig. 4 illustrates how SPEM work products are translated into XACDML elements. SPEM work products are artefacts consumed, produced, or modified by tasks. Roles perform tasks that use work products as input while producing other work products as output. The results of applying this algorithm can be seen in lines 3 and 5 in Fig. 2. The algorithm receives a DeliveryProcess as parameter (line 1). For each SPEM work product in the deliveryProcess (line 2), an XACDML temporary entity is created (line 3). Lines 4-6 are similar to the ones discussed in Fig. 3. We consider the initial dead state as the incoming one associated with the WBE of index "1" in the process WBS (Software production, in Fig. 1). The mapping algorithm of Fig. 4 identifies this initial dead state and creates a generate active state for it (lines 7-10). A generate active state creates temporary entities using a probabilistic distribution. In the process of Fig. 1, a generate active state that injects user stories into the initial dead state can be created. Not all systems need a generate active state. An alternative is configuring the initial quantity of user stories in the initial dead state. The tool we developed to support the mapping algorithms (see Section 5) allows the simulation analyst to include a generate active state in the XACDML model.

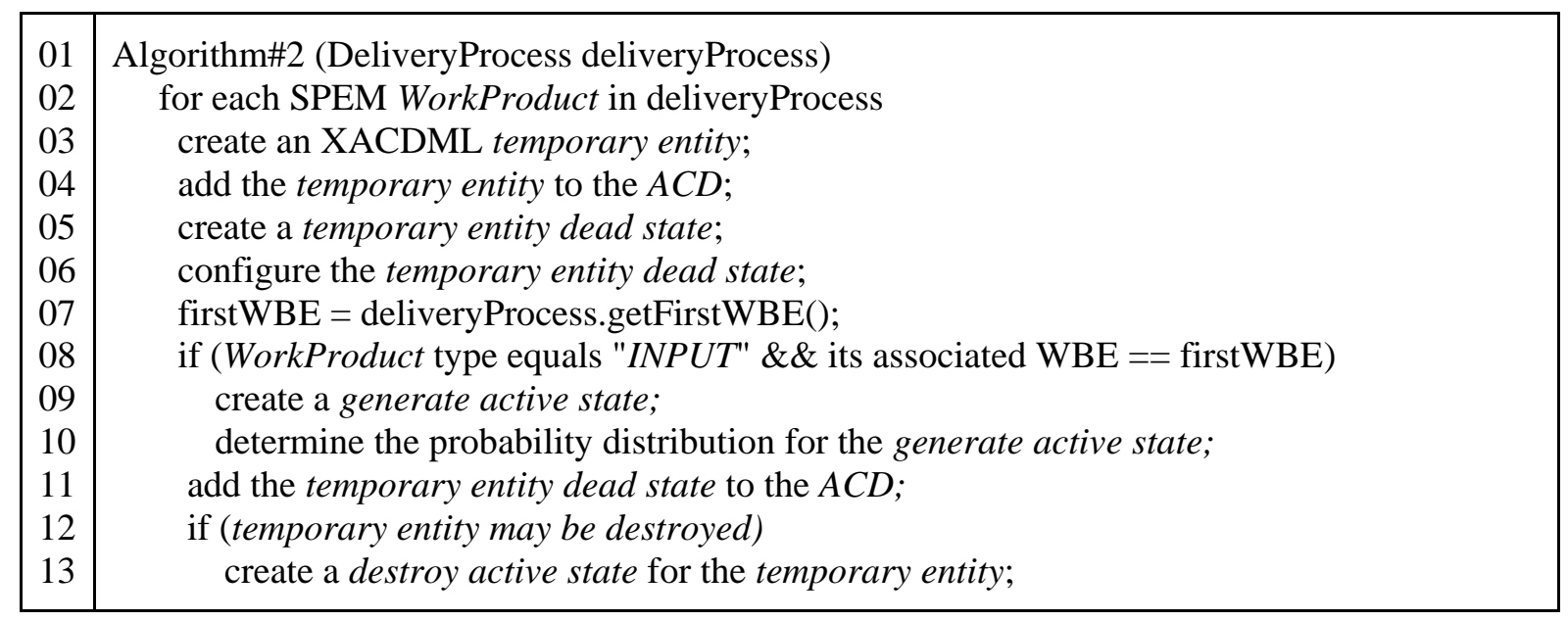

Figure 4: Mapping algorithm \#2 - "Maps SPEM work products to XACDML temporary entities, dead states, generate active states, and destroy active states".

When a temporary entity is not used any longer in the simulation, it can be destroyed. This is indicated in lines 12 and 13 of Fig. 4. We determine whether a temporary entity is destroyed based on which dead state it is located. In our example, a user story can only be destroyed if it is already implemented. This means that the temporary entity named User story must be in the XACDML dead state that was generated for the output work product of the "Pair programming with test driven development" task of Fig. 1. Although the algorithm detects which entities may be destroyed, the simulation analyst can decide not to destroy them at all for reporting and debugging purposes. The XACDML excerpt shown in Fig. 2 illustrates the XACDML tags generated by applying the algorithm of Fig. 4 with the decisions of not creating an XACDML generate active state for user stories and not destroying implemented user stories.

The algorithm presented in Fig. 5 is used for SPEM tasks and generates the XACDML excerpt exhibited on Fig. 2 (lines 07-16, and 25-34). A SPEM task describes an assignable unit of work to a role. In XACDML, the equivalent of a SPEM task is the concept of activity. The algorithm statement "create an XACDML activity;" in line 2 of Fig. 5 is translated, for example, into the <act id="Pair programming with test driven development" > tag on Fig. 2 (line 25). The value of the $i d$ attribute is derived from the task name.

We use quantitative data to configure the appropriate probability distribution and respective parameters for the duration of the active states (line 4 of Fig. 5). In our example, 
the duration of the activity "Pair programming with test driven development" is sampled from a lognormal distribution with parameters values of 1900 minutes for scale and 1200 minutes for shape. This is reflected in the element <stat type $=$ "LOGNORMAL" parml="1900" parm $2=" 1200 " />$ of line 26 in Fig. 2.

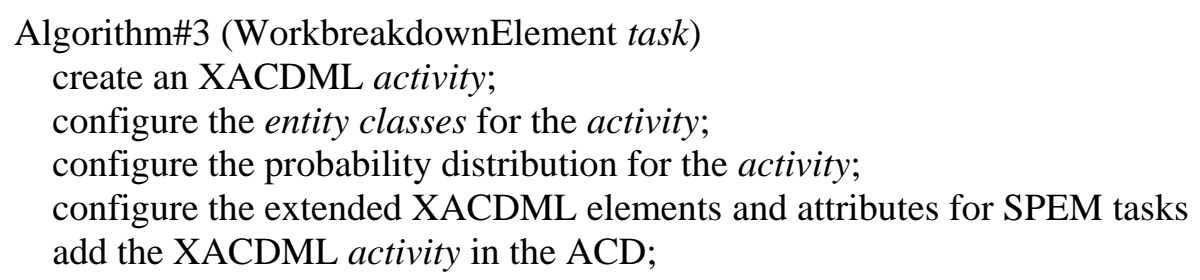

Figure 5: Mapping algorithm \#3 - "Maps SPEM tasks to XACDML activities".

An activity corresponds to an engagement of different type of entities, permanent and temporary. Both types carry information of their previous and next dead states as shown, for example, in lines 27 and 28 of Fig. 2. We created a new attribute for XACDML permanent entity classes named quantity_of_resources_needed. This attribute indicates the number of resources demanded to perform the activity. In our example, the "pair programming with test driven development" activity demands two idle resources to start servicing.

Line 5 of the mapping algorithm allows us to configure new elements and attributes of XACDML for SPEM support. The new element named spem (<spem>tag) consists of the mandatory attribute spem_type and the optional attributes parent, dependency_type, and condition_to_process. In addition to the new spem element, a new attribute named processing_quantity is used. When processing_quantity is set to "BATCH", it means all temporary entities in the incoming dead state are processed at once. If configured to "UNIT", only one is processed each time. The spem_type is used to configure the type of the WBE being mapped to XACDML (a task, in this case). The parent attribute allows identifying whether the task is nested inside other WBEs. Its value when set is used to discover the timebox of the parent WBE (we discuss the need for the timebox attribute in Section 5).

SPEM uses dependencies to define predecessor and successor relations among WBEs. The available dependency types are Finish-to-Start, Finish-to-Finish, Start-to-Start, and Start-to-Finish. The Finish-to-Start dependency type is by far the most common type of dependency [3] and the one we use in our implementation. Within our approach, the dependency_type and condition_to_process attributes are usually used together to provide better semantics for SPEM dependencies. When we set the dependency_type to "FINISH-TOSTART", we indicate that a WBE may only start when its predecessor element has finished. In our approach, a predecessor finishes in one of two ways; when one temporary entity or when a class of temporary entities is served by a predecessor active state. A class of temporary entities represents a set containing all temporary entities of certain type. The attribute named condition_to_process is used to establish a condition that must be satisfied in order to the XACDML active state starts servicing. Its possible values are "SINGLEENTITY-AVAILABLE" and "ALL-ENTITIES-AVAILABLE". The former value is the default behaviour for XACDML. The latter is an option in the extended XACDML. In our example, we set 29 temporary entities in the initial dead state (see line 05 of Fig. 2.). This number represents the initial size of the class of entities. In that way, since the active state "Pair programming with test driven development" was configured with dependency_type = "FINISH-TO-START" and condition_to_process = "ALL-ENTITIES-AVAILABLE", it can only start servicing if its predecessor active state ("Release Planning") has already processed all the 29 temporary entities. 
Fig. 6 illustrates the algorithm used for the creation of dummy active states (no simulation time is computed when running them). It is used to map SPEM milestones, iterations, activities, and phases to the extended XACDML. For a SPEM milestone, this algorithm is called once. The algorithm is called twice for phases, iterations, and activities, generating a pair of XACDML dummy active states that delimitates them in time. The first dummy active state of the pair represents the BEGIN of a phase, iteration, or activity. The second dummy active state indicates its END. This mapping algorithm is rather similar to the algorithm \#3 for tasks, with the difference that in addition to the wbe parameter of type WorkBreakdownElement, the algorithm also receives a parameter of type String that is used to name the dummy active state (line 1). Another difference resides in line 2 where only temporary entity classes are configured (we do not engage permanent entities in dummy active states). A third difference is the duration (line 4) that is set to zero, identifying it as an XACDML dummy active state. Line 6 adds the activity to the output XACDML.

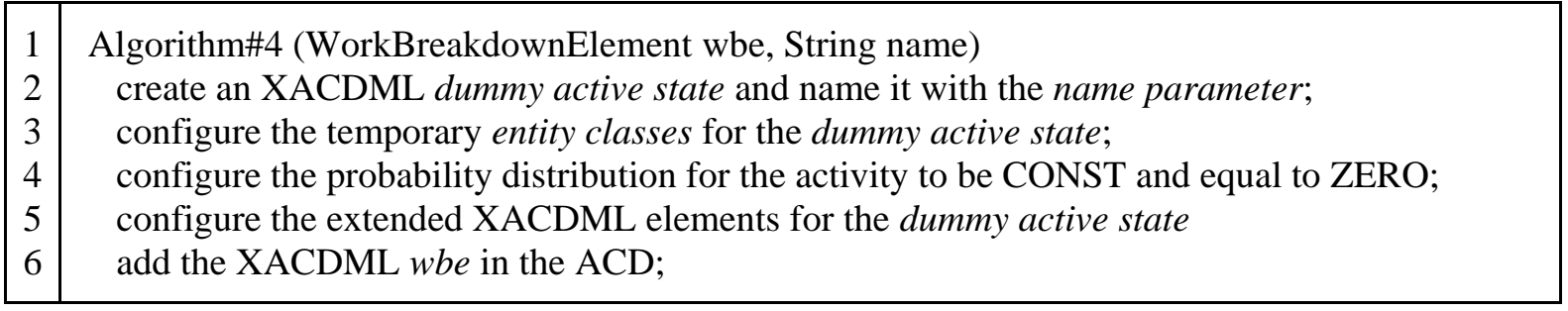

Figure 6: Mapping algorithm \#4 - "Maps SPEM iteration, activities, phases, and milestones to dummy active states with no simulation duration".

We use the recursive algorithm \#5 (Fig. 7) to generate all XACDML active states from SPEM WBEs. In the tree-like WBS structure for processes in SPEM, tasks and milestones are leaves whereas phases, iterations and activities are internal nodes that may have children.

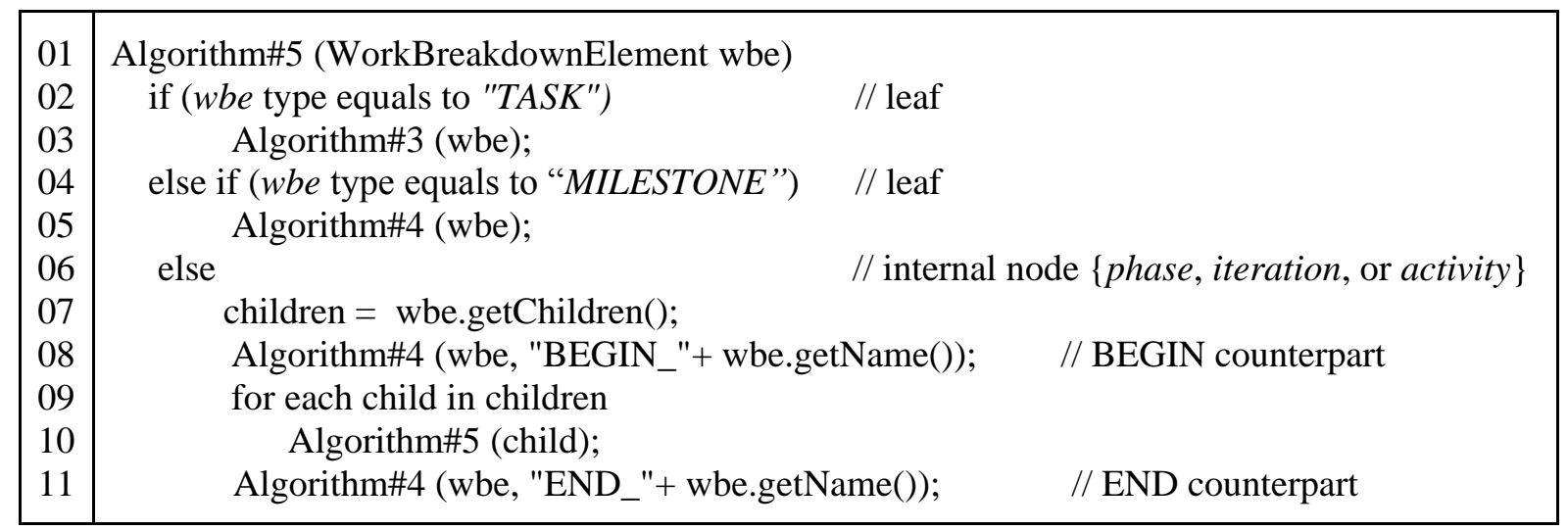

Figure 7: Recursive algorithm used to translate tasks, milestones, phases, iterations, and activities to the extended XACDML active states.

The algorithm receives as parameter an object of type WorkBreakDownElement (line 01). If this object is of type task or milestone (leaf), the algorithm \#3 or \#4 is called (lines 02-05). Otherwise, the wbe object is a phase, iteration, or activity (internal nodes with children), and two dummy active states must be created, one for BEGIN and another for END. The BEGIN dummy active state and the END dummy active state are created in line 08 and in line 11, by calling algorithm \#4. For each child in the wbe, a recursive call is made (line 10). When applied to our example, the execution of this algorithm generates lines 07-42 of the XACDML illustrated in Fig. 2. 


\section{TOOL SUPPORT}

We developed a tool that supports our approach and implements the mapping algorithms. The tool provides graphical capabilities to the simulation analyst and is compliant with SPEM and XACDML. It takes as input a SPEM process and translates it to an output XACDML simulation model.

Fig. 8 shows a SPEM process imported into our tool. The process is shown on the right side of the figure. In addition to support the mapping algorithms, the tool also provides the functionality of transforming the generated XACDML model into Java code that can be reused on-the-fly by the tool itself, allowing out of the box experimentation, without human intervention. On-the-fly Java code is generated by the use of eXtensible Stylesheet Transformation, XSLT. Gil and Hirata [5] also used XSLT, however, we adapted their transformations to support the XACDML extensions and to make the output Java code more reusable. The generated Java code uses a component library for the development of DES models [13]. The component library includes an executive that supports the three-phase worldview. We extended it, implementing support for the new elements and attributes of the extended XACDML.

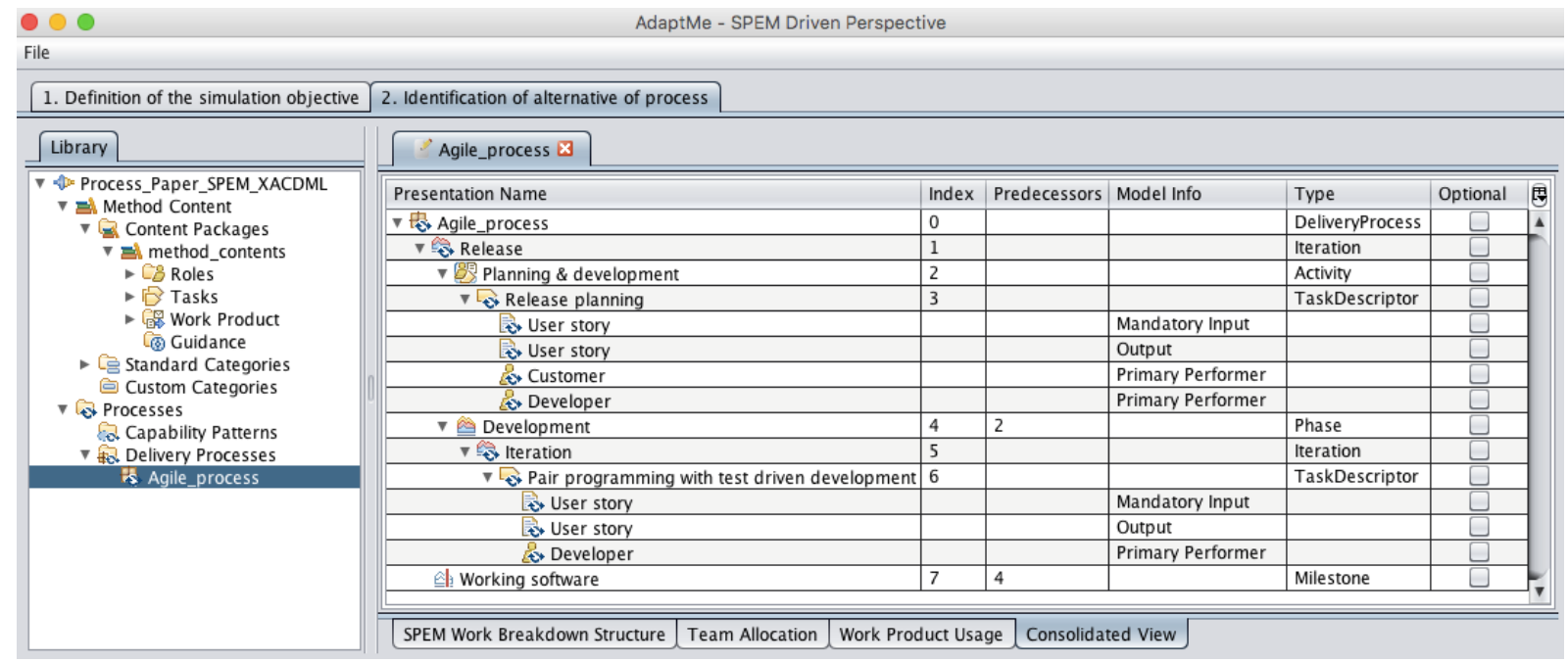

Figure 8: A SPEM process imported into our tool.

Fig. 9 shows a tool graphical support in which the duration of a SPEM task is configured with its corresponding probability distribution and parameters before generation of the extended XACDML model. In the lower part ("Extended XACDML attributes") we can use fields to configure all new XACDML elements and attributes. The fields are SPEM type sensitive. For instance, the field for "quantity of resources needed to perform a task" is only enabled when mapping SPEM tasks. The timebox field can only be configured for SPEM activities and iterations.

In what follows, we discuss the extensions to the simulation library [13]. In general they can be classified into extensions that establish conditions to the start of XACDML active states (evaluated at C-events) and extensions that implement the specific behaviour to be accomplished depending on the type of the SPEM WBE (executed at B-events).

One condition that must be evaluated before starting an active state is the condition configured with the attribute condition_to_process. As discussed, if this attribute is set to "ALL-ENTITIES-AVAILABLE", it specifies that the active state may only start when a class of entities has been produced (made available) by a predecessor active state. To make this possible, we define a counter for each object of type ActiveState. The counter is incremented 
in its B when temporary entities are processed. The increment is based on the value of the XACDML processing_quantity attribute. If it is set to "UNIT", the counter is incremented by one. If it is set to "BATCH", the counter is incremented by the number of entities within the batch. At the C-event of an active state, the counter of the previous active state is compared with the size of the class of entities. If they are equal, the previous active state has finished processing all entities in the class, what satisfies the partial condition to the start of the active state.

Although no simulation time is spent in each dummy active state of the pair generated from SPEM iterations and activities, we use their timebox as parameter. This parameter determines the moment the END counterpart of the pair must be scheduled in the simulation calendar. We implement this correct schedule by keeping record of the time the BEGIN counterpart has started. The END counterpart is scheduled at the simulation time resultant of the time the BEGIN counterpart has started plus the value presented in the timebox attribute. For instance, if a BEGIN_iteration dummy active state has started at simulation clock 480 and the iteration timebox is configured to 4800, the END_iteration counterpart will be scheduled to $5280(480+4800)$. It is important to note that no WBEs nested inside an iteration, or activity, may start servicing if the current clock plus estimated service time exceeds the current clock plus the timebox for its parent active state.

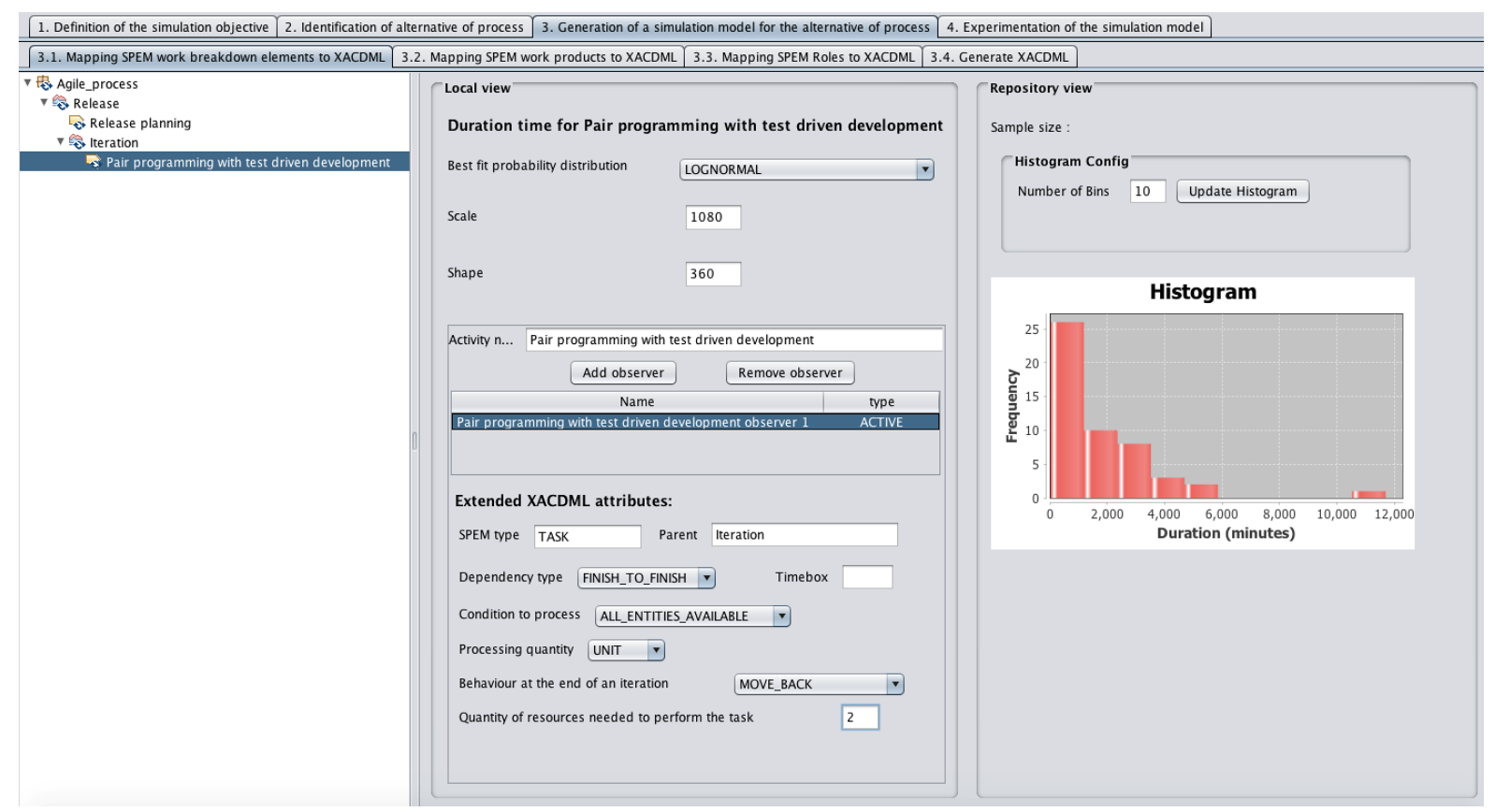

Figure 9: Graphical user interface developed to aid simulation analysts when mapping SPEM to extended XACDML.

In ACD, the default behaviour for an active state at its B event is to release resources and entities back to their respective dead states. Our approach adds additional behaviour depending on the type of the SPEM WBEs represented by the spem_type attribute. SPEM activity, phase, and iteration represent a period of time in a project with start and finish times. At the B events of the BEGIN and END active states representing these WBEs, we record the times they started and finished, respectively. We also increment a second counter that is used to know how many times these SPEM WBEs were performed in the simulated process.

At the B event of the dummy active state representing the end of an SPEM iteration, in addition to the logic describe above, one of two other behaviours is possible: (i) move all remaining entities in intermediary dead states to the initial dead state or (ii) keep the current status of the intermediary dead states. These options can be configured by the use of the new 
XACDML attribute named iteration_behaviour. The initial dead state of an iteration is the incoming one associated with the BEGIN dummy active state generated for the SPEM iteration. Intermediary dead states are all remaining ones presented within the limits of the BEGIN and END dummy active states for the iteration. As a side effect of executing the logic at the $\mathrm{B}$ event of an iteration, the counter presented in every single active state used to determine the number of temporary entities produced by the active state is set to zero and the number of temporary entities in the class of entities is set to the new quantity in the initial dead state of the iteration.

We decide to not consider SPEM phase as time boxed. Instead, its end is determined when a class of temporary entities is processed by the END counterpart active state (dummy) generated when mapping a SPEM phase to extended XACDML. A milestone represents a specific moment in time within the project. Within our approach, a milestone is reached when a class of temporary entities is processed. Hence, at a B-event of a milestone, we record the time it is reached.

\section{EXAMPLE}

In this section, we use an example of a software development project that enacts a typical agile method as the process defining the work to be accomplished by a team of developers. We use our tool in this example to demonstrate its applicability.

The process is the same presented in Fig. 1. This example was chosen since it is analogous to a case study that uses real project data presented by Melis et al. [6]. Their work does not use phases and milestones as in our example. We include these elements in order to cover all SPEM WBE elements during experimentation and verify if the mappings are correct. We argue that this does not affect the results, since phase and milestone are dummy active states and, hence, their executions do not contribute to the total time of the simulation run.

The quantitative model elements were taken from their work either directly or indirectly, i.e. derived from the results presented by the authors. The results presented in their work are compared with ours to validate the generated simulation model. We use the following parameters and values (in parentheses): number of user stories (29), number of developers (4), typical release duration (30 days), time spent in release planning meeting (1 day). Melis et al. use a hybrid simulation model with DES and SD in which user stories are implemented by incremental sessions of development. In their work, the duration of the development sessions is no longer than four hours. Within a development session, one of the following implementation tasks is performed: pair programming with test-driven development, debugging, or refactoring. Since we focus on DES, we had to derive the parameters for the duration of a "Pair programming with test driven development" task to complete one discrete unit of a user story (not a portion of it in an incremental way at the end of each development session). We estimate the duration distribution being lognormal with parameters of mean = 1900 days and standard deviation $=1200$ days (the work of Melis et al. describes the effort to produce user stories as being lognormal, what helps us during this process of parameters estimation). We made several adjustments before getting to the values used in the example. For simplicity purpose, we assume that the duration to implement a user story by "Pair programming with test driven development" task includes the time spent in debugging and refactoring activities.

We, with the aid of our tool, performed a number of simulation runs using the input parameters discussed. We inspected the trace reports and log files generated during the simulation runs for verification purposes, attempting to identify logical flaws in the model. The simulation output was compared with those taken from the work of Melis et al. in order to validate our model. The results of the simulation runs are shown in Table II. As can be 
seen, our results agree well with the results presented in the work of Melis et al. and the actual data of the reference system. We report here our results using the same number of simulation runs used in their work. The output analysis of the simulation results is out of our scope. Refer to their work for details.

Table II: Comparison between simulation results averaged over 200 runs of Melis et al. [6] and our work. Standard deviations are reported in parentheses.

\begin{tabular}{|l|c|c|c|}
\hline \multicolumn{1}{|c|}{ Output measure } & Real data & Simulation (Melis et al.) & Our model \\
\hline Days & 60 & $61.9(13.5)$ & $58.1(5.4)$ \\
\hline User stories & 29 & $28.2(1.6)$ & $28.8(0.3)$ \\
\hline Number of releases & 2 & $2.5(0.6)$ & $1.9(0.5)$ \\
\hline Iterations per release & 3 & $2.7(0.3)$ & $2.9(0.4)$ \\
\hline
\end{tabular}

\section{DISCUSSIONS}

In this work, we propose a model-driven approach to automate the generation of DES models of process models. To achieve our goal, we extended XACDML, the XSLT transformations from XACDML to Java, and a DES simulation library. All extensions were necessary in order to cope with process specific elements described in SPEM, including hierarchical structures that are not explicitly defined in ACD, and consequently, XACDML.

Our approach is materialised in a tool that allows generating simulation models and tailoring the generation process to fit analysts' interests. Instead of generating Java code from SPEM processes in a single step, we focus our attention in using an intermediate notation (XACDML) for verification purposes. We argue it is much easier to verify XML files (XACDML) representing ACD models than verifying code written in a programming language.

We cover all SPEM WBEs that can be used for the construction of the WBS that represents a SPEM process. In SPEM, these WBEs can be further configured by additional attributes (isRepeatable, hasMultipleOccurrences, isOngoing, isEventDriven, and isOptional). We support all these attributes. The isOptional attribute is of particular interest since it allows creating conditional flows within a process. It indicates a WBE within the WBS whose inclusion is not mandatory when performing a project, since it depends on some domain specific condition(s). The simulation analyst can configure a WBE as optional and indicate the condition that must be satisfied for its execution during mapping of SPEM to XACDML. One such condition could indicate, for example, that a deployment to production task is only executed when a minimum number of user stories have been implemented. The conditions must be expressed by the use of some pre-defined variables representing the simulation clock, quantity of entities in the incoming dead state, quantity of entities produced by the previous activity, among others.

SPEM can be used to model any process of the systems engineering field [3, 7]. We verified this flexibility in our approach testing other examples not related to software development. In these examples we verified the generated XACDML simulation models looking for inconsistencies. In particular, we verified the correct mapping of all XACDML elements discussed in this paper, including conditional flows (testing several conditional branches). Likewise, we checked for each example, the XACDML model assumption that the specifications of the destinations of entities and resources were all made in an unequivocal manner. No problem was found during the verifications. We also conducted several runs with additional examples, verifying the simulation results and log files generated for each run in order to identify possible logical flaws. No error was found, what makes us to believe that our 
approach with tool support is feasible and has the potential to support any process modelled with the SPEM specification.

The closest work to our current research [2] proposes an approach to derive a hybrid simulation model (DES and SD) from a SPEM 1.1 process model. We consider the hybrid mechanism as an advantage when contrasted to our work. By using their approach, the intervention of the stakeholders in developing the simulation model is reduced, but not as much when compared to our work. Since their work was made with an earlier specification of SPEM (version 1.1), it could not benefit from the main improvement in SPEM 2.0, that is, the clear separation of method content and its application in processes [3] - page 11. As a consequence of the lack of concern separation, their approach requires modelling using UML use cases and activity diagrams to model process work sequence. The need to modelling imposes some burden in order to transform the models. Our work is SPEM 2.0 compliant and hence, benefits from this separation of concerns, not requiring this additional and manual step of UML modelling. We only demand a simulation analyst with ACD knowledge to set the parameters during mapping of SPEM to XACDML.

We consider that our approach is effective and efficient. It is effective since it mitigates the effort needed to build simulation models for processes by automating the generation. It is efficient, since the time spent in conducting experiments is limited to the time a simulation analyst needs to configure the mappings of SPEM to XACDML.

Basic knowledge of ACD notations and XACDML is needed in order to use our approach. This is one drawback of our work. Although the mapping algorithms presented allow the automation of the translation process, a simulation analysts is needed to configure the mappings with appropriate parameters. For instance, the simulation analyst must decide whether it is necessary or not to include an XACDML generate active state during the mapping process.

MDD provides new capabilities for the development of simulation models, but during development of our approach, we found difficult adopting MDD, since it is restricted to particular modelling languages and requires a steep learning curve with respect to its principles and methods.

The decomposition of work products is an important feature of SPEM processes where one input work product of a WBE is decomposed into one or more output work products of the same or different types. XACDML should be extended to support this feature. The work of Hirata and Paul [14] can be used with this purpose. We intend to embrace this feature within our approach in a near future.

\section{CONCLUSION}

In this work, we proposed a model-driven approach to automate the generation of DES models of process models. We strived to reduce the need of human intervention. There is no need of knowledge of some programming language in order to use our approach.

The results obtained and presented in this paper indicate that transforming SPEM models to XACDML models is a feasible choice. However, we acknowledge that the approach still needs to be validated and improved with more examples and case studies in industry. We are interested in dynamic definition of engineering processes during enactment. As a future work, we will extend it to enable the analysis of the impact of process changes during project evolution. The idea is to support the decision-making of what process changes to conduct in an ongoing project. The decision is taken by evaluating several process alternatives with the use of simulation after evaluating the impact of these alternatives on output variables of interest in the project. 


\section{ACKNOWLEDGEMENT}

CAPES (Coordenação de Aperfeiçoamento de Pessoal de Nível Superior) supported this work.

The work of the second author was supported by the Conselho Nacional de Desenvolvimento Científico e Tecnológico under the grant number Universal 01/2016 403921/2016-3 and the grant number PQ 303666/2015-3.

\section{REFERENCES}

[1] Ali, N. B.; Petersen, K.; Wohlin, C. (2014). A systematic literature review on the industrial use of software process simulation, Journal of Systems and Software, Vol. 97, 65-85, doi: $10.1016 / j . j s s .2014 .06 .059$

[2] Park, S.; Choi, K.; Yoon, K.; Bae, D.-H. (2007). Deriving software process simulation model from SPEM-based software process model, Proceedings of the $14^{\text {th }}$ Asia-Pacific Software Engineering Conference, 382-389

[3] Object Management Group. Software \& Systems Process Engineering Meta-Model Specification, from http://www.omg.org/spec/SPEM/, accessed on 19-06-2017

[4] Pidd, M. (2004). Computer Simulation in Management Science, $5^{\text {th }}$ edition, Wiley, New York

[5] Gil, J. N.; Hirata, C. M. (2003). XACDML - Extensible ACD Markup Language, Proceedings of the $36^{\text {th }}$ Annual Symposium on Simulation, 343-350

[6] Melis, M.; Turnu, I.; Cau, A.; Concas, G. (2006). Evaluating the impact of test-first programming and pair programming through software process simulation, Software Process: Improvement and Practice, Vol. 11, No. 4, 345-360, doi:10.1002/spip.286

[7] Ruiz-Rube, I.; Dodero, J. M.; Palomo-Duarte, M.; Ruiz, M.; Gawn, D. (2013). Uses and applications of software \& systems process engineering meta-model process models. A systematic mapping study, Journal of Software: Evolution and Process, Vol. 25, No. 9, 9991025, doi:10.1002/smr.1594

[8] Çetinkaya, D.; Verbraeck, A.; Seck, M. D. (2015). Model continuity in discrete event simulation: A framework for model-driven development of simulation models, ACM Transactions on Modeling and Computer Simulation, Vol. 25, No. 3, Paper 17, 24 pages, doi:10.1145/2699714

[9] Hollmann, D. A.; Cristiá, M.; Frydman, C. (2015). CML-DEVS: A specification language for DEVS conceptual models, Simulation Modelling Practice and Theory, Vol. 57, 100-117, doi:10.1016/j.simpat.2015.06.007

[10] Kim, S.; Sarjoughian, H. S.; Elamvazhuthi, V. (2009). DEVS-suite: A simulator supporting visual experimentation design and behavior monitoring, Proceedings of the Spring Simulation Multiconference (SpringSim 2009), Paper 161, 7 pages

[11] Bergero, F.; Kofman, E. (2010). PowerDEVS: a tool for hybrid system modeling and real-time simulation, Simulation, Vol. 87, No. 1-2, 113-132, doi:10.1177/0037549710368029

[12] Arias, R.; Hirata, C. M. (2011). Mapping of software model to simulation model for performance requirement specification, Proceedings of the $44^{\text {th }}$ Annual Simulation Symposium, 142-150

[13] Araújo Filho, W. L.; Hirata, C. M. (2004). Translating activity cycle diagrams to Java simulation programs, Proceedings of the $37^{\text {th }}$ IEEE Annual Symposium on Simulation, 157-164

[14] Hirata, C. M.; Paul, R. J. (1996). Object-oriented programming architecture for simulation modelling, International Journal in Computer Simulation, Vol. 6, No. 2, 269-287 